\title{
Coefficients for the Derivation of Posterior and Right Sided Chest Leads From the 12-lead Electrocardiogram
}

\author{
Michael R Jennings ${ }^{1}$, Ali S Rababah ${ }^{1}$, Pardis Biglarbeigi ${ }^{1}$, Rob Brisk ${ }^{1}$, Daniel Güldenring ${ }^{2}$, \\ Raymond Bond $^{1}$, James McLaughlin ${ }^{1}$, Dewar D Finlay ${ }^{1}$ \\ ${ }^{1}$ Ulster University, Newtownabbey, UK \\ ${ }^{2}$ HTW Berlin, Berlin, Germany
}

\begin{abstract}
Background: The 12-lead ECG is spatially limited in diagnosing cardiac abnormalities. Additional leads (right sided and posterior leads) are inconvenient in a clinical setting, however, they can be derived. In this paper we report on the development of coefficients to allow derivation of right sided and posterior leads.

Method: Analysis was performed using body surface potential maps (BSPM) recorded from 910 patients in two centres. Recordings were made up of healthy controls $(n=314)$, peak balloon inflation during elective percutaneous coronary angioplasty $(n=88)$, myocardial infarction $(n=271)$ and left-ventricular hypertrophy $(n=237)$. All recordings were expanded to the 352-node Dalhousie torso. Coefficients to allow derivation of right sided and posterior leads were generated by linear regression. Further coefficients from a previously reported study were used for performance comparisons.

Results: Correlation coefficients between recorded and derived leads were significantly improved using the new coefficients $(p<0.05)$ in leads V7-V12.

Conclusion: We have developed coefficients that allow the derivation of 10 additional leads from the 12-lead ECG.
\end{abstract}

\section{Introduction}

The 12-lead ECG remains the most important tool in the diagnosis of cardiac abnormalities [1]. This method has a spatial resolution across the torso that results in high specificity in anomaly detection $[2,3]$. However, it has been shown that more leads can improve the detection accuracy of some cardiac abnormalities $[4,5]$. It is inconvenient to use lead systems that use additional recording sites in a clinical environment, especially posterior leads underneath a supine patient. One solution for this is to derive the additional leads from the information already present in the existing 12-lead ECG. Derived leads are less accurate than measured leads [6], however, they still have potential to improve diagnostic accuracy [7]. Body surface potential maps (BSPM) capture potentials across the entire torso and therefore allow the development of transformation coefficients that in turn allow the derivation of additional lead sets from existing lead systems [8]. In this study, we aim to introduce coefficients toward the derivation of additional right-sided (V3R-V6R) and posterior chest leads (V7-V12) from the 12-lead ECG. This problem has been tackled by researchers in the past however our work involves the use of more extensive data sets in the development of the derivation coefficients. In our study we show how our method compares to previous work. We have included the transformation coefficients as derived in our work.

\section{Method}

\subsection{Data}

The data were recorded from two centres, with a total of 910 recordings. The data were recorded using a similar electrode configuration of 117 thoracic unipolar leads recorded with respect to the Wilson Central Terminal (WCT). Limb lead data were also recorded for each subject. Recordings from both centres were expanded to a 352-node Dalhousie torso [9] using Laplacian interpolation. Each recording was a single beat in length sampled at $500 \mathrm{~Hz}$. Data were split at random to $75 \%$ training $(n=685)$ and $25 \%$ test $(\mathrm{n}=225)$.

Centre one data $(n=176)$ has already been described [10]. Recordings were from patients undergoing elective percutaneous transluminal coronary angioplasty (PTCA). Two recordings from each patient were taken: one during rest and the other during peak balloon inflation (PBI). Inflations were carried out in one of three coronary arteries: LAD ( $n=32), \operatorname{LCX}(n=22), \operatorname{RCA}(n=34)$. Rest and PBI recordings were kept together during training/test partition. Centre two data $(n=734)$ was described previously 
$[11,12]$. Recordings were from patients experiencing myocardial infarction $(\mathrm{n}=271)$, left ventricular hypertrophy $(\mathrm{n}=237)$ and healthy controls $(\mathrm{n}=226)$.

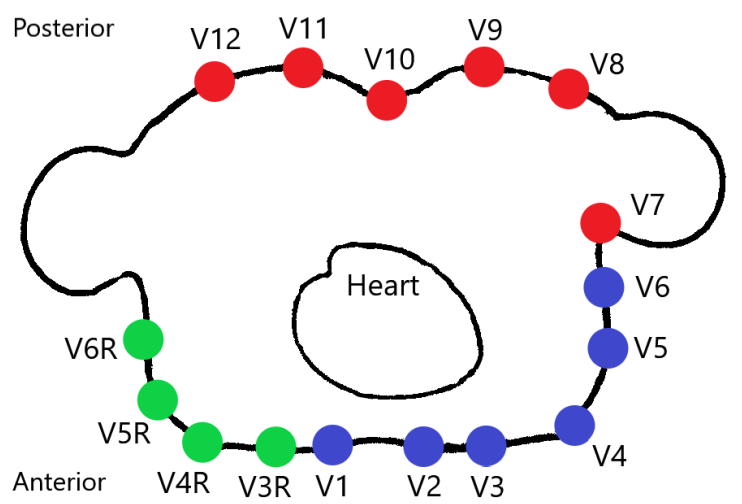

Figure 1. Cross section (plan view) of the thorax with approximate locations of precordial leads (blue), posterior leads (red) and right-sided leads (green)

\subsection{Coefficient Generation}

Eight of the independent channels of the 12-lead ECG were extracted from each recording (I-II, V1-V6). The leads to be derived were also extracted. The leads to be derived consisted of the commonly recognised posterior leads (V7-V12) and right-sided precordial leads (V3RV6R). The approximate locations of these leads are shown in Figure 1.

Recorded leads and the leads to be derived were used in the coefficient generation. All recordings in the training set $(n=685)$ were pooled prior to calculation. Linear regression was then used to derive the transform coefficients as follows:

$$
\beta=\left[\left(R L_{\text {train }}^{T} \cdot R L_{\text {train }}\right)^{-1} R L_{\text {train }}^{T}\right] \cdot D L_{\text {train }}
$$

Where $\beta$ is the resulting $8 \times 10$ matrix of coefficients that relates recorded and derived leads. $R L_{\text {train }}$ represents an $m_{\text {train }} \mathrm{x} 8$ matrix of recorded leads (I-II, V1-V6) taken from the training dataset. $D L_{\text {train }}$ represents an $m_{\text {train }} \mathrm{x} 10$ example of derived leads (V7-V12, V3R-V6R) also taken from the training dataset. In all of our experiments, both $R L_{\text {train }}$ and $D L_{\text {train }}$ were made up of pooled data from the corresponding respective leads from all subjects in our training set. $m_{\text {train }}$ indicates the total number of ECG samples in the training set $(n=218,094)$

\subsection{Lead Derivation}

The derived leads were generated using the calculated coefficients on the test dataset $(n=226)$. The leads were derived using (2).

$$
\hat{D L_{\text {test }}}=R L_{\text {test }} \cdot \beta
$$

Where $\hat{D L} L_{\text {test }}$ was an $m_{\text {test }} \mathrm{x} 10$ matrix containing the estimate of the derived leads (V7-V12, V3R-V6R). $R L_{\text {test }}$ was an $m_{\text {test }} \mathrm{x} 8$ matrix of recorded leads (I-II, V1-V6) taken from the test dataset. $\beta$ was the $8 \times 10$ matrix of derivation coefficients as defined in (1). $m_{\text {test }}$ indicates the total number of ECG samples in the test set $(n=69,909)$

\subsection{Verification}

The recorded leads of the test dataset were used to benchmark performance. The Pearson correlation coefficient (CC) and root-mean square error (RMSE) were calculated by comparing the recorded leads (previously extracted from our BSPM data) ( $x$ ) with our derived equivalents $(y)$. CC is calculated as follows:

$$
\rho_{(x, y)}=\frac{1}{M-1} \sum_{m=1}^{M}\left(\frac{\overline{y_{m}-\mu_{y}}}{\sigma_{y}}\right)\left(\frac{x_{m}-\mu_{x}}{\sigma_{x}}\right)
$$

Where $\rho_{(x, y)}$ is the CC. $x$ and $y$ represent the recorded leads $\left(R L_{\text {test }}\right)$ and derived leads $\left(\hat{D} L_{\text {test }}\right)$ respectively. $M$ indicates the number of samples, $\mu$ is the mean, $\sigma$ is the standard deviation and $m$ is the sample number. The RMSE between recorded and derived lead was calculated using Equation (4):

$$
\operatorname{RMSE}_{(x, y)}=\sqrt{\frac{1}{M} \sum_{m=1}^{M}\left(x_{m}-y_{m}\right)^{2}}
$$

\section{Results}

\subsection{Generated Coefficients}

The coefficients were arranged in an $8 \times 10$ matrix as shown in Table 1. The rows represent the recorded leads (I-II, V1-V6) with columns representing the derived leads (V7-V12, V3R-V6R). Both CC and RMSE were calculated for each derived lead. The median values are displayed in Table 1.

Figures 2 and 3 show the CC and RMSE values for each derived chest lead respectively. The error-bar plot indicates the median value (circle) with the $25^{\text {th }}$ and $75^{\text {th }}$ interquartile ranges (whiskers).

\subsection{Analysis}

Previously published coefficients from Nagenthiraja et al. [13] were used to verify the derivation performance. The previously published coefficients transform the 12lead ECG to posterior leads V7-V12 using coefficients calculated from a multiple regression model. Figure 4 shows 
Table 1. Derived lead coefficients $(\beta)$ and their calculated performance

\begin{tabular}{|c|c|c|c|c|c|c|c|c|c|c|c|}
\hline & \multicolumn{10}{|c|}{ Derived Leads } \\
\hline & & $V 7$ & $V 8$ & $V 9$ & $V 10$ & $V 11$ & $V 12$ & $V 3 R$ & $V 4 R$ & $V 5 R$ & $V 6 R$ \\
\hline \multirow{10}{*}{ 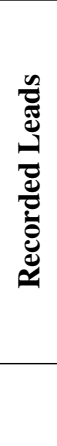 } & $I$ & -0.0063 & -0.0597 & $\begin{array}{l}-0.1912 \\
\end{array}$ & -0.2975 & -0.3655 & -0.4039 & $\begin{array}{l}-0.2030 \\
\end{array}$ & $\begin{array}{l}-0.3340 \\
\end{array}$ & $\begin{array}{l}-0.3809 \\
\end{array}$ & -0.4015 \\
\hline & $I I$ & -0.0397 & -0.0515 & -0.0398 & -0.0415 & -0.0495 & -0.0460 & 0.1331 & 0.1686 & 0.1570 & 0.1424 \\
\hline & $V 1$ & -0.1374 & -0.1765 & -0.1997 & -0.1819 & -0.1189 & -0.0567 & 0.8030 & 0.5037 & 0.3217 & 0.1848 \\
\hline & $V 2$ & -0.0348 & -0.0268 & -0.0174 & -0.0197 & -0.0204 & -0.0341 & -0.1878 & -0.1401 & -0.0916 & -0.0657 \\
\hline & $V 3$ & 0.0887 & 0.0723 & 0.0631 & 0.0663 & 0.0562 & 0.0716 & 0.1678 & 0.1051 & 0.0434 & 0.0320 \\
\hline & $V 4$ & -0.0192 & -0.0077 & -0.0220 & -0.0463 & -0.0499 & -0.0618 & -0.0812 & -0.0320 & 0.0050 & 0.0017 \\
\hline & $V 5$ & -0.2299 & -0.2715 & -0.2482 & -0.1774 & -0.0918 & -0.0408 & 0.0291 & 0.0109 & -0.0216 & -0.0432 \\
\hline & $V 6$ & 0.7940 & 0.7156 & 0.5638 & 0.3126 & 0.1279 & 0.0568 & -0.0380 & -0.0439 & -0.0238 & 0.0055 \\
\hline & $C C$ & 0.98 & 0.96 & 0.94 & 0.91 & 0.95 & 0.97 & 0.99 & 0.97 & 0.96 & 0.95 \\
\hline & $R M S E$ & $46.2 \mu \mathrm{V}$ & $51.8 \mu V$ & $48.3 \mu \mathrm{V}$ & $38.6 \mu \mathrm{V}$ & $27.0 \mu \mathrm{V}$ & $23.7 \mu \mathrm{V}$ & $25.8 \mu V$ & $35.3 \mu \mathrm{V}$ & $31.6 \mu \mathrm{V}$ & $28.8 \mu \mathrm{V}$ \\
\hline
\end{tabular}

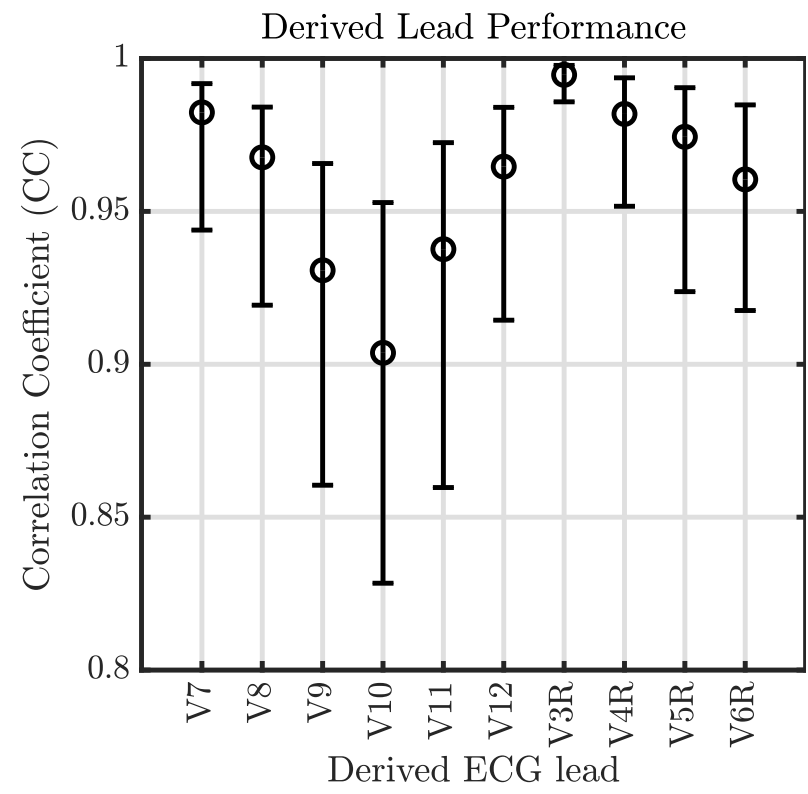

Figure 2. Correlation coefficient between recorded and derived chest leads

a box plot comparing the coefficients introduced in this study with those previously suggested. Median (centre), interquartile ranges (box edges) and extremes (whisker) are displayed for both sets of coefficients. A Wilcoxon signed-rank test was used to indicate statistical difference between results. Significant improvements in $\mathrm{CC}(\mathrm{p}<0.05)$ were noted in V7-V12.

For derived posterior leads (V7-V12), the CC decreases toward V10. This may be due to the increasing distance between recorded and derived lead positions on the torso. The same is true for derived right-sided chest leads (V3RV6R) which show CC inversely proportional to distance from the recorded leads. CC increases in V11 and V12. These leads are almost opposite the recorded leads V2-

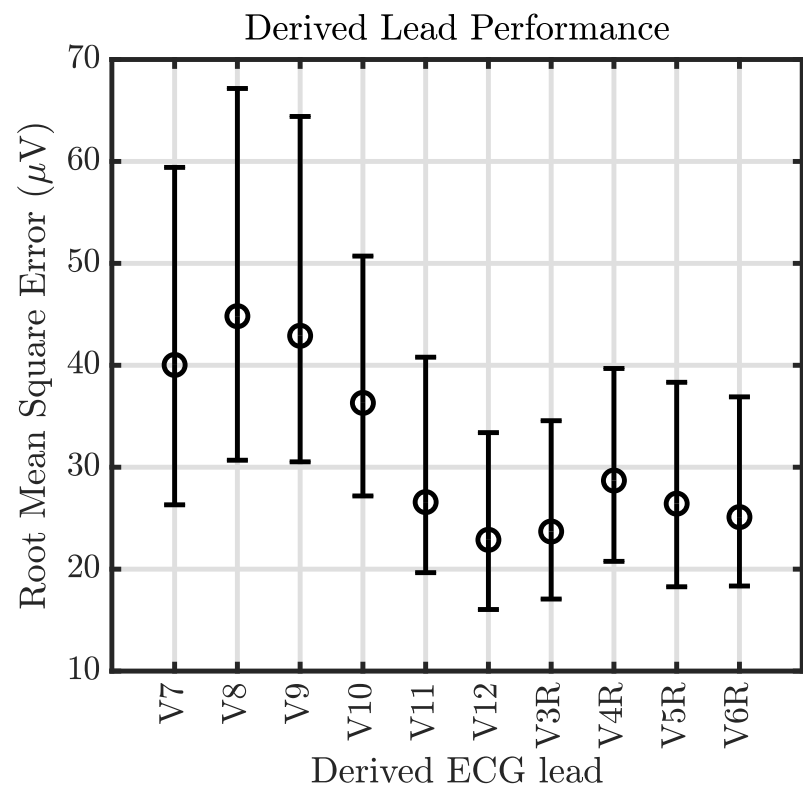

Figure 3. Root mean square error between recorded and derived chest leads

V4 across the thorax, potentially making them pseudoinverse. This may have increased the accuracy of the derived coefficients. RMSE does not follow the same pattern as CC. RMSE is proportional to the recorded lead amplitude. Lower potentials present in leads more distal from the heart may have made RMSE appear lower compared to leads more proximal to the heart.

\section{Conclusion}

We have provided coefficients toward the derivation of posterior and right-sided chest leads (V7-V12; V3R-V6R) from the 12-lead ECG using linear regression. Median correlation between recorded and derived leads was greater 


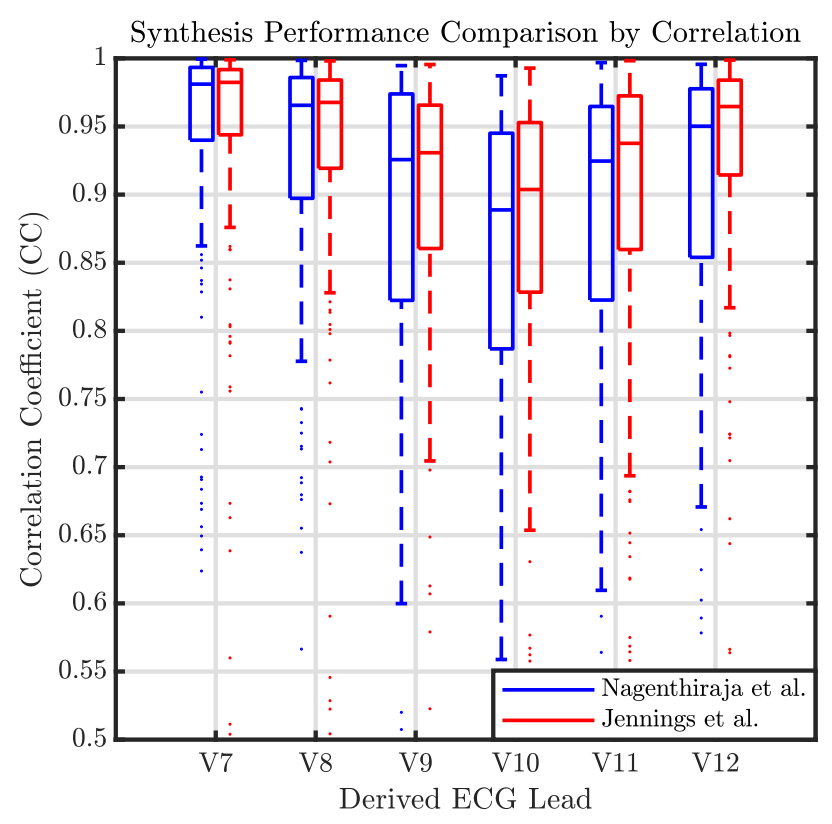

Figure 4. Box plot of CC comparing previously published coefficients with the those introduced in this study

than 0.9 for all channels. This method showed statistically significant improvements in the generation of leads V7-V12 when compared to a previous study. An imbalanced number of recordings in each centre and lack of data may have reduced the performance of regression calculations. Furthermore, to our knowledge there are no published right-sided precordial lead coefficients to compare against. Further studies will be required to investigate the efficacy of additional-lead coefficients to cardiac abnormality detection.

\section{Acknowledgements}

This project is part of the Eastern Corridor Medical Engineering centre (ECME). It is supported by the European Union's INTERREG VA Programme, managed by the Special EU Programmes Body (SEUPB).

\section{References}

[1] Thygesen K, Alpert JS, Jaffe AS, Chaitman BR, Bax JJ, Morrow DA, White HD. Fourth universal definition of myocardial infarction. European Heart Journal 2018; 24(3):107-138. ISSN 26187620.

[2] Perron A, Lim T, Pahlm-Webb U, Wagner GS, Pahlm O. Maximal increase in sensitivity with minimal loss of specificity for diagnosis of acute coronary occlusion achieved by sequentially adding leads from the 24-lead electrocardiogram to the orderly sequenced 12-lead electrocardiogram. Journal of Electrocardiology 2007;40(6):463-469. ISSN 00220736.
[3] Dharmaprani D, Lahiri A, Ganesan AN, Kyriacou N, McGavigan AD. Comparative spatial resolution of 12-lead electrocardiography and an automated algorithm. Heart Rhythm 2020;17(2):324-331. ISSN 15563871.

[4] Ornato JP, Menown IB, Peberdy MA, Kontos MC, Riddell JW, Higgins GL, Maynard SJ, Adgey J. Body surface mapping vs 12-lead electrocardiography to detect ST-elevation myocardial infarction. American Journal of Emergency Medicine 2009;27(7):779-784. ISSN 07356757.

[5] Trägårdh E, Claesson M, Wagner GS, Zhou S, Pahlm O. Detection of acute myocardial infarction using the 12-lead ECG plus inverted leads versus the 16-lead ECG (with additional posterior and right-sided chest electrodes). Clinical Physiology and Functional Imaging 2007;27(6):368-374. ISSN 14750961.

[6] Saitoh Y, Goseki Y, Yazaki Y, Yamashina A. Pitfall of synthesized posterior/right-sided chest lead electrocardiograms. Journal of Arrhythmia 2012;27:PJ2_064. ISSN 1880-4276.

[7] Ashida T, Tani S, Nagao K, Yagi T, Matsumoto N, Hirayama A. Usefulness of synthesized 18-lead electrocardiography in the diagnosis of STEMI. American Journal of Emergency Medicine 2017;35(3):448-457. ISSN 15328171 .

[8] Guldenring D, Finlay DD, Bond RR, Kennedy A, McLaughlin J, Galeotti L, Strauss DG. The derivation of the spatial QRS-T angle and the spatial ventricular gradient using the Mason-Likar 12-lead electrocardiogram. Journal of Electrocardiology 2015;48(6):1045-1052. ISSN 15328430

[9] Horáček BM. Numerical model of an inhomogeneous human torso. Advanced Cardiology 1974;10(51).

[10] Horáček BM, Warren JW, Penney CJ, MacLeod RS, Title LM, Gardner MJ, Feldman CL. Optimal electrocardiographic leads for detecting acute myocardial ischemia. Journal of Electrocardiology dec 2001;34(4):97-111. ISSN 00220736.

[11] Kornreich F Montague T J RPM. Body surface potential mapping of ST segment changes in acute myocardial infarction: Implications for ECG enrollment criteria for thrombolytic therapy. Circulation 1993;87(3):773-782. ISSN 00097322.

[12] Schijvenaars BJ, Kors JA, van Herpen G, Kornreich F, van Bemmel JH. Interpolation of body surface potential maps. Journal of Electrocardiology 1995;28:104-109. ISSN 00220736.

[13] Nagenthiraja K, Andersen M P, Struijk J. Linear model for deriving the posterior ECG leads V7-V12 in healthy subjects - Project Library, Aalborg University. Ph.D. thesis, Aalborg Universitet, Aalborg, 2008.

Address for correspondence:

Michael Jennings

NIBEC, Ulster University

NEWTOWNABBEY, United Kingdom

jennings-m5@ulster.ac.uk 